\title{
Ethical Issues in Pediatric Psychiatric Clinical Trials
}

\section{Robb A and Engle $K^{\star}$}

Children's National Health System, Washington DC, USA

\begin{abstract}
Background: Over the last decade, the number of research trials investigating psychiatric medications for children and adolescents has increased dramatically. However, this research brings to light ethical concerns regarding the doubly vulnerable population of children with psychiatric disorders. This article summarizes some of the practices established to reduce the risks this population may incur from participating in clinical trials research.
\end{abstract}

Methods: A narrative synthesis was developed after a review of several summary articles focused on ethical issues in pediatric psychiatric clinical trials.

Results: Federal regulations have been established to ensure that the rights and safety of children are protected during research participation, such as the four levels of risk to which every research protocol is assigned. This assignment is made based on all of the potential risks and associated with participation. Safeguards exist to ensure risks are minimized or eliminated whenever possible. The consent process and the requirement of assent (if applicable) is an opportunity to ensure that legal guardians and participants alike understand the meaning of their participation in the research and do not expect to receive the same as one would receive from standard medical care. All studies are required to receive approval from an Investigational Review Board before starting any study-related procedures, and some studies receive additional oversight from data safety monitoring boards if they involve a highly vulnerable population, such as pediatric psychiatric clinical trials.

Conclusions: Even with safeguards like these in place, many concerns still remain regarding the ethics surrounding this area of research. However, the promise of research to inform clinical care - when conducted ethically and carefully makes it worthwhile for the research community to take on the challenging ethical issues posed by pediatric psychiatric clinical trials.

Keywords: Research ethics; Children and adolescents; Informed consent; Psychiatry; Clinical trials

\section{Introduction}

Over the last decade, the number of research trials investigating psychiatric medications for children and adolescents has increased dramatically. Previously, pediatric use was based on data from clinical trials in adults [1]. However, adults differ from children and adolescents significantly, including physical development, metabolism, effects and side effects $[2,3]$. Therefore, the uninformed clinical use of these medications on a pediatric population could be viewed as a large-scale, uncontrolled experiment. Given the differences between adults and children and adolescents, it would be naive to believe that medications are effective and safe for youth simply because they are effective for adults, and it would be irresponsible to act on this assumption. Fortunately, researchers and practitioners have acknowledged the paucity of data for psychopharmacological treatments for pediatric psychiatric disorders and have implemented clinical trials designed to provide the necessary data [2]. This research also brings to light the unique ethical concerns of doing research with the doubly vulnerable population of children with psychiatric disorders. It is important to provide the data necessary to safely and effectively treat pediatric patients with psychopharmacological agents, but it is imperative that this research be conducted ethically.

\section{Materials and Methods}

The purpose of this article is to inform readers about the practices that have been established in order to reduce the risks children and adolescents with psychiatric disorders may incur from participating in clinical trials research. MD Consult was searched for review articles using keywords related to pediatrics, psychiatric clinical trials, and ethical issues or concerns. From these results, twelve review articles were thoroughly reviewed, and nine of these were selected to serve as sources of information for this summary article.

\section{Results}

A population is deemed as "vulnerable" in a research context when it does not possess the full capacity to consent to research participation freely and knowingly and is at risk for being exploited [1]. Children and adolescents, therefore, are considered to be vulnerable research populations since they are not legally able to make decisions for themselves and may or may not be of a developmental capacity to do so. A psychiatric disorder further reduces this decision-making ability, leaving youth with a psychiatric diagnosis "doubly vulnerable". It is the responsibility of researchers and guardians to determine whether it is acceptable for a child to participate in a trial. Federal regulations have been established to ensure that the rights and safety of children are protected during research participation [3].

The federal government has determined four levels of risk for research protocols. The level of risk of a trial determines the authorizations to be acquired during the informed consent process. Trials under the first level of risk are those that pose no more than

*Corresponding author: Krista Engle, Children's National Health System, Washington DC, USA, Tel: 202-476-3910; Fax: 202-476-5898; E-mail: kengle@childrensnational.org

Received December 20, 2013; Accepted January 07, 2014; Published January 09, 2014

Citation: Robb A, Engle K (2014) Ethical Issues in Pediatric Psychiatric Clinical Trials. Health Care Current Reviews 2: 115. doi: 10.4172/2375-4273.1000115

Copyright: ( 2014 Robb A, et al. This is an open-access article distributed under the terms of the Creative Commons Attribution License, which permits unrestricted use, distribution, and reproduction in any medium, provided the original author and source are credited. 
minimal risk to the participant, or no more than would be experienced by the patient in daily life. These trials are not required to provide benefit to the participant, and the permission of one parent and the assent of the child (if applicable) must be obtained. In the second level of risk, trials pose greater than minimal risk to the participants, but they also present the prospect of direct benefit to the individual. In these situations, the more-than-minimal risk is justified by the anticipated benefit to the participant. Therefore, these trials must obtain permission from one parent and the child's assent to enroll a child in the study, although the child's lack of assent can be overridden. The third level of risk encompasses studies that pose greater than minimal risk to participants with no prospect of direct benefit to them. Because risk outweighs benefit, permission for participation must be obtained from both parents in addition to the child's assent. These same requirements must be met by studies that fall under the fourth level of risk, which involves studies that pose greater than a minor increase above minimal risk with no prospect of direct benefit $[1,3,4]$.

In order to classify studies under one of the four levels, their potential risks and benefits must be assessed. Pediatric psychiatric clinical trials may offer several potential benefits to participants. Participants may receive direct benefit from being treated with an effective medication or therapy offered in the therapy arm of a study. If patients are randomized to the placebo arm of a randomized controlled trial (RCT), they may not be receiving direct benefit from the drug being tested, but this does not mean that they are receiving no benefit from participating in the study. Many participants in RCTs exhibit a "placebo effect," or some helpful or therapeutic change in response to administering a placebo $[5,6]$. A participant's conviction that they are on a medication often improves the symptoms they exhibit. This seems to be especially true for children with depressive disorders, as Parellada et al. observed. In their article on placebo effect and pediatric psychiatric trials, the authors state that "the degree of placebo response (not the drug response) is the single most powerful predictor of drug superiority versus placebo in pediatric antidepressant studies" [5]. Many studies include regular check-up or monitoring visits, which provides the participant with routine care from a psychiatrist. The amount children and adolescents benefit from "therapeutic contact" may be dependent on other variables such as age. Rutherford et al. observed that the benefits of therapeutic contact are positively correlated with patient age in pediatric depression trials. Because participation in pediatric research is time-consuming, clinical trials frequently provide some form of compensation, usually monetary compensation [6]. The amount of compensation provided considers the family's travel, related expenses and time, but excessive amounts may be coercive for families.

Of course, there are several risks posed by pediatric clinical research. A medication being tested may not be effective, or well tolerated, causing adverse reactions. If confidentiality is breached, a participant's diagnosis could be revealed to others, and participants may experience stigma or discrimination because of this. Even if the child or adolescent is present for the consent and assent process, he or she may agree to participate without fully understanding the purpose of the research or the risks they are undertaking by participating in the trial. Children and adolescents may also feel pressured by their parents or other authority figures to participate in a clinical trial without thoroughly understanding what his or her participation warrants.

Because these hazards exist in this type of research, certain safeguards are built into the research process to ensure that these inherent risks are minimized or eliminated whenever possible. One safeguard is the consent process. This process should "ideally be a dynamic process of information sharing between participant or guardian and researcher" rather than merely the signing of a document [1]. The discussion that takes place should help the parents or guardians and the participant make a fully informed decision regarding their participation in the study. Some researchers are concerned that the informed consent process has become overly burdensome and that the documents include too much legal language, making them incomprehensible for their audience. The purpose of the consent discussion is to make sure that the participant and his or her caregivers understand what participation in a specific research protocol entails. Therefore, it is important that the terminology used during the consent process is comprehensible for someone unfamiliar with the research, and it should also be suitable for a young audience if the participant's assent is required.

The researcher should use this opportunity to make sure that neither guardians nor participants believe that participating in a clinical trial will warrant the same benefits as receiving standard medical care. This phenomenon is known as the "therapeutic misconception" [4]. The aims of clinical research and medical care are far from the same; research is conducted to benefit the whole of society by contributing to general scientific knowledge, whereas medical care's purpose is to benefit a sole individual. This difference is clear to someone who designs and implements clinical trials on a regular basis, but it may not be as obvious to someone that has never participated in research or who is seeking a new, effective treatment option. Hoop notes that "the desperation and hope experienced by parents of suffering children may make them particularly susceptible to this misconception regarding their children's research participation" [1]. Therefore, it is even more critical that researchers conducting pediatric psychiatric clinical trials take the time during the informed consent process to ensure that participants and their guardians understand that their participation in the research may potentially benefit them, but does not hold the same promise as receiving medical care.

Under certain circumstances, it is necessary to acquire assent, another safeguard used in pediatric research, during the informed consent process. Assent is a child's affirmative agreement to participate in research. This means that it is not enough for a child to simply not object to participation. It must be evident that the child understands what their participation requires and firmly agrees to participate to say that one has obtained assent $[3,4]$. To ensure that a child or adolescent fully understands what providing their assent means, it is critical that they play an equal role in the informed consent process and that the discussion is brought down to a level that they can fully comprehend. To determine whether a child is capable of providing assent, researchers generally follow the "Rule of 7s," which uses a child's developmental capacity to determine whether it is necessary to acquire their assent. This rule assumes that children under the age of 7 normally do not have the capacity to assent, that children between the ages of 7 and 14 have the capacity to assent, and that children older than 14 have the capacity to participate in the informed consent process [4]. This rule is generally followed in pediatric research. However, the clinical judgment of the researcher interacting with a potential participant and enrolling him or her in a particular trial may be of greater importance than this general rule when determining whether assent should be required. This should be the case if, in the researcher's opinion, not following the "Rule of $7 \mathrm{~s}$ " will help ensure that the participant and their family do what is in their best interests [4].

A third safeguard utilized to minimize risks to child and adolescent participants in psychiatric clinical trials is the oversight of certain clinical trials by an outside data safety monitoring board. All research 
studies, regardless of their topic or procedures, must be approved by an Investigational Review Board before enrolling participants, and federal regulations require some studies that involve highly vulnerable populations to have ongoing oversight by specialized committees called data safety monitoring boards (DSMBs) [1]. These independent review boards monitor the progress of the study to ensure timelines are met, risk is minimized, no safety signals arise, and that the study is conducted ethically and in accordance with FDA or other regulatory guidelines [7]. These bodies are composed of experts in the topic of the research and others with a variety of perspectives on the research process, and the constituents of a board are autonomous from the researcher and the sponsor of the study to ensure that its decisions are non-biased [8]. DSMBs follow each study under their oversight from protocol implementation to the end of data analysis, and they have the authority to require a research team to halt study procedures for one of several reasons. First, the DSMB may stop a study early if an interim analysis of the data shows a greater degree of separation between drug and placebo than was originally desired. Therefore, the endpoint is reached early, and there is no need to continue the study. The group may stop a study early after a futility analysis shows that the endpoint will not be reached after the total planned number of study subjects has completed the study. DSMBs may also halt trials when a safety signal arises such as the excessive weight gain on an antipsychotic in a treatment of early onset schizophrenia study (TEOSS) or when the ability to recruit the requisite number of subjects falls behind the recruitment time lines [9].

Even with these safeguards in place, many concerns still remain regarding the ethics surrounding pediatric psychiatric clinical trials. Although national regulations have been created to guide ethical practices, it is often noted that different institutions and researchers variably interpret these regulations, making it difficult to guarantee that every research protocol is held to the same ethical standards. Questions arise regarding "mature minors" who are clearly capable of making decisions for them, but regulations requiring parental consent may prevent them from participating in research that could provide them with direct benefits [4]. Research is constantly branching in new directions (i.e. genetic testing research), and these new directions inevitably raise ethical questions with which the research community has not had to deal [3]. The responsibility of researchers to protect their participants is heightened in the case of pediatric psychiatry research, but this should not discourage researchers from exploring the issues that need to be addressed in order to provide the best care possible for children and adolescents with psychiatric disorders. The promise of research to inform clinical care-when conducted ethically and carefully - makes it worthwhile for the research community to take on the challenging ethical issues posed by pediatric psychiatric clinical trials.

\section{Acknowledgements}

Dr. Adelaide Robb and Krista Engle are with Children's National Health System in Washington, DC.

\section{References}

1. Hoop J, Smyth A, Roberts $L$ (2008) Ethical issues in psychiatric research on children and adolescents. Child Adolesc Psychiatr Clin N Am 17: 127-148.

2. McVoy M, Findling R (2009) Child and adolescent psychopharmacology update. Psychiatr Clin North Am 32: 111-133.

3. Laventhal N, Tarini B, Lantos $\mathrm{J}$ (2012) Ethical issues in neonatal and pediatric clinical trials. Pediatr Clin North Am 59: 1205-1220.

4. Chen D, Shepherd L (2009) When, why, and how to conduct research in child and adolescent psychiatry: Practical and ethical considerations. Psychiatr Clin North Am 32: 361-380

5. Parellada M, Moreno C, Moreno M, Espliego A, de Portugal E, et al. (2012) Placebo effect in child and adolescent psychiatric trials. Eur Neuropsycho Pharmacol 22: 787-799.

6. Rutherford B, Sneed J, Tandler J, Rindskopf D, Peterson B, et al. (2011) Deconstructing pediatric depression trials: An analysis of the effects of expectancy and therapeutic contact. J Am Acad Child Adolesc Psych 50: 782 795.

7. Leon A (2012) Independent data and safety monitoring in psychiatric intervention research. J Clin Psychiatry 73: 257-263.

8. Carandang C, Santor D, Gardner D, Carrey N, Kutcher S (2007) Data safety monitoring boards and other study methodologies that address subject safety in 'high-risk' therapeutic trials in youths. J Am Acad Child Adolesc Psych 46: 489-492.

9. Sikich L, Frazier J, McClellan J, Findling RL, Vitiello B, et al. (2008) Doubleblind comparison of and second-generation antipsychotics in early onset schizophrenia and schizo-affective disorder: 